\title{
Every 3-connected, locally connected, claw-free graph is Hamilton-connected
}

Armen Asratian

The self-archived postprint version of this journal article is available at Linköping University Institutional Repository (DiVA):

http:/ / urn.kb.se/ resolve?urn=urn:nbn:se:liu:diva- 143760

N.B.: When citing this work, cite the original publication.

Asratian, A., (1996), Every 3-connected, locally connected, claw-free graph is Hamilton-connected, J ournal of Graph Theory, 23(2), 191-201. https:// doi.org/ 10.1002/ (SICI)1097-

0118(199610)23:2\&lt;191::AID-J GT10\&gt;3.0.CO;2-K

Original publication available at:

https:// doi.org/ 10.1002/ (SICI)1097-0118(199610)23:2\&tt;191::AID-

JGT10\&gt;3.0.CO;2-K

Copyright: Wiley (12 months)

http://eu.wiley.com/WileyCDA/ 


\title{
Every 3-Connected, Locally Connected, Claw-Free Graph is Hamilton-Connected
}

\author{
A. S. Asratian
}

DEPARTMENT OF MATHEMATICS, UNIVERSITY OF UMEA S-901 87 UMEÅ, SWEDEN DEPARTMENT OF MATHEMATICAL CYBERNETICS

YEREVAN STATE UNIVERSITY

YEREVAN 375049, REPUBLIC OF ARMENIA

\section{ABSTRACT}

A graph $G$ is locally connected if the subgraph induced by the neighbourhood of each vertex is connected. We prove that a locally connected graph $G$ of order $p \geq 4$, containing no induced subgraph isomorphic to $K_{1,3}$, is Hamilton-connected if and only if $G$ is 3connected. (C) 1996 John Wiley \& Sons, Inc.

\section{INTRODUCTION}

We use [1] for terminology and notation not defined here and consider finite simple graphs only. Let $V(G)$ and $E(G)$ denote, respectively, the vertex set and edge set of a graph $G$. For each vertex $u$ of $G$, the neighbourhood $N(u)$ is the set of all vertices adjacent to $u$ and $M(u)=N(u) \cup\{u\}$. If $W$ is a nonempty subset of $V(G)$, then we denote by $\langle W\rangle$ the subgraph of $G$ induced by $W$. A graph $G$ is called claw-free if $G$ has no induced subgraph isomorphic to $K_{1,3}$.

A graph $G$ is said to be hamiltonian if it has a cycle containing all the vertices of $G$. A path with end vertices $x$ and $y$ is called an $(x, y)$-path. An $(x, y)$-path is a Hamilton path of $G$ if it contains all the vertices of $G$. A graph $G$ is Hamilton-connected if every two vertices $x, y$ are connected by a Hamilton $(x, y)$-path.

The following concept of local connectivity was defined in [4]: A graph $G$ is locally $n$ connected, $n \geq 1$, if $\langle N(u)\rangle$ is $n$-connected for each $u \in V(G)$. Later, Oberly and Sumner [8] proved that every connected, locally connected, claw-free graph $G$ with $|V(G)| \geq 3$ is hamiltonian. Clark [6] improved this result by showing that in a graph $G$ satisfying 
the Oberly-Sumner condition, each vertex of $G$ lies on a cycle of every length from 3 to $|V(G)|$ inclusive.

When is a locally connected, claw-free graph Hamilton-connected? This problem was investigated first by Chartrand, Gould and Polimeni [3]. They proved that a connected, locally 3-connected, claw-free graph is Hamilton-connected. Later, Kanetkar and Rao [7] improved this result, by showing that the condition of 3-connectedness can be changed to 2-connectedness. Moreover, they proved that even in this case each pair of distinct vertices $x$ and $y$ of $G$ is connected by a path of every length from $d(x, y)$ to $|V(G)|-1$ inclusive.

In this paper we give a complete solution of the problem. We prove that a locally connected, claw-free graph $G$ with $|V(G)| \geq 4$ is Hamilton-connected if and only if $G$ is 3 -connected. This result was conjectured by Broersma and Veldman [2].

\section{NOTATIONS AND PRELIMINARY RESULTS}

Let $P$ be a path of $G$. We denote by $\vec{P}$ the path $P$ with a given orientation and by $\overleftarrow{P}$ the path $P$ with the reverse orientation. If $u, v \in V(P)$, then $u \vec{P} v$ denotes the consecutive vertices of $P$ from $u$ to $v$ in the direction specified by $\vec{P}$. The same vertices, in reverse order, are given by $v \bar{P} u$. We use $w^{+}$to denote the successor of $w$ on $\vec{P}$ and $w^{-}$to denote its predecessor. We assume that an $(x, y)$-path $\vec{P}$ has an orientation from $x$ to $y$. We will denote by $k(G)$ and $\alpha(G)$ the connectivity and the independence number of a graph $G$, respectively. Let $H$ be a graph with $V(H)=A \cup\{u, v\}$ where $\langle A\rangle$ is a complete subgraph of $H$ and $u z, v z \in E(H)$ for each $z \in A$. In this situation we let $u[A] v$ denote a Hamiltonian $(u, v)$-path of $H$.

Proposition 2.1. Let $G$ be a connected, locally connected, claw-free graph, and $u$ a vertex of $G$. If there exist two non-adjacent vertices $z_{1}, z_{2} \in N(u)$ such that $N(u) \cap N\left(z_{1}\right) \cap$ $N\left(z_{2}\right)=\emptyset$, then the sets $A_{1}=\left\{z_{1}\right\} \cup\left(N\left(z_{1}\right) \cap N(u)\right)$ and $A_{2}=\left\{z_{2}\right\} \cup\left(N\left(z_{2}\right) \cap N(u)\right)$ have the following properties:

(1) $A_{1} \cup A_{2}=N(u), A_{1} \cap A_{2}=\emptyset$ and $\left|A_{i}\right| \geq 2$ for $i=1,2$.

(2) The graphs $H_{1}=\left\langle A_{1}\right\rangle$ and $H_{2}=\left\langle A_{2}\right\rangle$ are complete and there exists an edge $v_{1} v_{2}$ where $v_{1} \in A_{1}$ and $v_{2} \in A_{2}$.

Proof. Clearly, $A_{1} \cap A_{2}=\emptyset$. If $A_{1} \cup A_{2} \neq N(u)$ then there is a vertex $z_{3} \in N(u)$ such that $z_{3} z_{1}, z_{3} z_{2} \notin E(G)$ and the set $\left\{z_{1}, z_{2}, z_{3}, u\right\}$ induces $K_{1,3} ;$ a contradiction. So $A_{1} \cup A_{2}=N(u)$. If one of the graphs $H_{1}$ and $H_{2}$, say $H_{1}$, contains two nonadjacent vertices $s$ and $t$ then the set $\left\{u, s, t, z_{2}\right\}$ induces $K_{1,3}$; a contradiction. So, $H_{1}$ and $H_{2}$ are complete graphs. The connectedness of $\langle N(u)\rangle$ implies that there exists an edge $v_{1} v_{2}$ where $v_{1} \in A_{1}$ and $v_{2} \in A_{2}$. Then $d(u)>2,\left|A_{1}\right| \geq 2$ and $\left|A_{2}\right| \geq 2$.

Proposition 2.2. Let $G$ be a connected, locally connected, claw-free graph, and let $u$ be a vertex of $G$. Furthermore, let $w$ be a cut vertex of $H=\langle N(u)\rangle$. Then the following properties hold:

(1) The graph $H-w$ has two components and each of them is a complete graph.

(2) The graph $H$ has at most two cut vertices. Moreover, if $H$ has two cut vertices then they are adjacent. 
Proof. The first property follows from the fact that $G$ is claw-free. Let $H_{1}$ and $H_{2}$ be components of $H-w$. Then for some $i \in\{1,2\}$, say $i=1, w$ is adjacent to all the vertices of $H_{i}$. Since $w$ is a cut vertex of $H$, we can deduce that $z_{1}=w$ for each edge $z_{1} z_{2} \in E(G)$ with $z_{1} \in V\left(H_{1}\right) \cup\{w\}, z_{2} \in V\left(H_{2}\right)$. This means that $H$ has at most two cut vertices. Moreover, $H$ has two cut vertices if and only if $w$ is adjacent to only one vertex from $V\left(H_{2}\right)$. This vertex is the second cut vertex of $H$.

Proposition 2.3. Let $G$ be a connected, locally connected, claw-free graph. If $v \in N(u)$ and $v$ is not a cut vertex of $H=\langle N(u)\rangle$ then there is a Hamilton $(u, v)$-path of $\langle M(u)\rangle$.

Proof. If $H$ has no cut vertex then $2 \leq k(H)$. Since $G$ is claw-free, $\alpha(H) \leq 2$. Hence, by a theorem of Chvatál and Erdös [5], $H$ is hamiltonian. This implies that there exists a Hamilton $(u, v)$-path of $\langle M(u)\rangle$.

If $H$ has a cut vertex $w$, then by Proposition 2.2, $H-w$ has two components and each of them is a complete graph. Since $v$ is not a cut vertex, the existence of a Hamilton $(u, v)$-path of $\langle M(u)\rangle$ is evident.

Let $z$ be an internal vertex of an $(x, y)$-path $P, x \neq y$. We say that $P$ has a local detour of $z$ if there exists a path in $\langle N(z) \backslash\{x, y\}\rangle$ with origin outside $P$ and terminus a neighbour of $z$ on $P$. The following result was obtained in [6].

Proposition 2.4 [6]. Let $G$ be a claw-free graph with $|V(G)| \geq 3$ and $P$ be an $(x, y)$-path of length $n, x \neq y, 3 \leq n \leq|V(G)|-2$. If $P$ has a local detour then $G$ contains an $(x, y)$-path $Q$ of length $n+1$ with $V(P) \subset V(Q)$.

Theorem 2.5. Let $G$ be a connected, locally connected, claw-free graph and $x, y$ be two distinct vertices of $G$. If there exists an $(x, y)$-path of length at least 3 including the set $N(x) \cup N(y)$ then there exists a Hamilton $(x, y)$-path of $G$.

Proof. It is sufficient to prove that if $P$ is an $(x, y)$-path of length $n<|V(G)|-1$ and $N(x) \cup N(y) \subseteq V(P)$ then there exists an $(x, y)$-path $Q$ of length $n+1$ with $V(P) \subset V(Q)$.

Let $P=x_{0} x_{1} \cdots x_{n}$, where $x_{0}=x$ and $x_{n}=y$. Since $G$ is connected and $n<$ $|V(G)|-1$, the set $U=\cup_{i=1}^{n-1} N\left(x_{i}\right) \backslash V(P)$ is not empty. If $P$ has a local detour at $x_{j}$ for some $1 \leq j \leq n-1$ then, by Proposition 2.4, there exists an $(x, y)$-path $Q$ of length $n+1$ such that $V(P) \subset V(Q)$. Assume now that

(1) for each $j=1, \ldots, n-1, P$ has no local detour at $x_{j}$.

Consider a vertex $v \in U$. Since $G$ is claw-free, $x_{j-1} x_{j+1} \in E(G)$ for each $x_{j} \in N(v) \cap$ $V(P)$. Let $i_{1}=\min _{x_{i} v \in E(G)} i$ and $u_{1} u_{2} \cdots u_{r}$ be a shortest $\left(v, x_{1+i_{1}}\right)$-path in the graph $\left\langle N\left(x_{i_{1}}\right)\right\rangle$, where $u_{1}=v$ and $u_{r}=x_{1+i_{1}}$. Since $G$ is claw-free, $r \leq 4$. Furthermore, since $N(x) \cup N(y) \subseteq V(P)$, (1) implies that $r \geq 4$. So, $r=4, u_{3} \in\left\{x_{0}, x_{n}\right\}$ and $u_{2} \in$ $V(P) \backslash\left\{x_{0}, x_{n}\right\}$.

Let $u_{2}=x_{i_{2}}$ for some $i_{2}, 1 \leq i_{1}<i_{2} \leq n-1$.

Case 1. $u_{3}=x_{0}$. We have $x_{i_{2}} x_{1+i_{2}}, v x_{i_{2}}, x_{i_{2}} x_{0} \in E(G)$ and $v x_{0}, v x_{1+i_{2}} \notin E(G)$. Then $x_{1+i_{2}} x_{0} \in E(G)$, because $G$ is claw-free. If $i_{1}=1$ then there is an $(x, y)$-path $Q$ of length $n+1$, where $Q=x_{0} x_{i_{2}} v x_{1} x_{2} \cdots x_{i_{2}-1} x_{i_{2}+1} \cdots x_{n}$. Let $i_{1} \geq 2$ that is $v x_{1} \notin E(G)$. Since $G$ is claw-free, $E(G) \cap\left\{x_{1} x_{1+i_{1}}, x_{1} x_{1+i_{2}}, x_{1+i_{1}} x_{1+i_{2}}\right\} \neq \emptyset$. Hence there exists an $(x, y)$-path $Q$ of length $n+1$ where

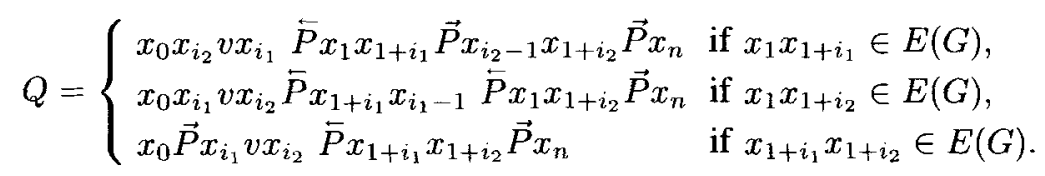


Case 2. $u_{3}=x_{n}$. If $v x_{n-1} \in E(G)$ then $Q=x_{0} \vec{P} x_{i_{1}} v x_{n-1} \stackrel{\leftarrow}{P} x_{1+i_{1}} x_{n}$ is the $(x, y)$-path of length $n+1$.

Let $v x_{n-1} \notin E(G)$. Then $i_{2}<n-1$ and we have $x_{i_{j}} x_{i_{j}-1}, v x_{i_{j}}, x_{n} x_{i_{j}} \in E(G)$ and $v x_{n}, v x_{i_{j}-1} \notin E(G)$ for $j=1,2$. This implies $x_{n} x_{i_{1}-1}, x_{n} x_{i_{2}-1} \in E(G)$ because $G$ is claw-free. We have now that $x_{n} x_{n-1}, x_{n} x_{i_{1}-1}, x_{n} x_{i_{2}-1} \in E(G)$. Therefore $E(G) \cap$ $\left\{x_{n-1} x_{i_{1}-1}, x_{n-1} x_{i_{2}-1}, x_{i_{1}-1} x_{i_{2}-1}\right\} \neq \emptyset$ since $G$ is claw-free. Then $G$ has an $(x, y)$-path $Q$ of length $n+1$, where

$$
Q= \begin{cases}x_{0} \vec{P} x_{i_{1}-1} x_{i_{1}+1} \vec{P} x_{i_{2}-1} x_{n-1} \stackrel{\leftarrow}{P} x_{i_{2}} v x_{i_{1}} x_{n} & \text { if } x_{n-1} x_{i_{2}-1} \in E(G) \\ x_{0} \vec{P} x_{i_{1}-1} x_{n-1} \stackrel{\leftarrow}{P} x_{i_{2}+1} x_{i_{2}-1} \stackrel{P}{P} x_{i_{1}} v x_{i_{2}} x_{n} & \text { if } x_{n-1} x_{i_{1}-1} \in E(G) \\ x_{0} \vec{P} x_{i_{1}-1} x_{i_{2}-1} \stackrel{P}{P} x_{i_{1}} v x_{i_{2}} \vec{P} x_{n} & \text { if } x_{i_{1}-1} x_{i_{2}-1} \in E(G)\end{cases}
$$

Theorem 2.6. Let $u, v$ be two distinct vertices of a 3-connected, locally connected, clawfree graph $G$ with $d(u, v) \leq 2$. If $N(v) \cap N\left(w_{1}\right) \cap N\left(w_{2}\right) \neq \emptyset$ for each pair of non-adjacent vertices $w_{1}, w_{2} \in N(v)$ then there exists a Hamilton $(u, v)$-path of $G$.

Proof. Taking Theorem 2.5 into consideration, it is sufficient to prove that there exists a $(u, v)$-path $Q$ with $N(u) \cup N(v) \subseteq V(Q)$. First we prove that there is a $(u, v)$-path $P$ with $N(u) \subseteq V(P)$. Let $H=\langle N(u)\rangle$.

Case 1. $d(u, v)=2$. Since $G$ is 3-connected, by a theorem of Whitney [9], there are three $(u, v)$-paths $Q_{1}, Q_{2}, Q_{3}$ such that $Q_{i}=u P_{i} v,\left|V\left(P_{i}\right) \cap N(u)\right|=1, i=1,2,3$, and $P_{1}, P_{2}, P_{3}$ are vertex disjoint. If $V\left(P_{i}\right) \cap N(u)=\left\{u_{i}\right\}$ for $i=1,2,3$ then, by Proposition 2.2 , one of the vertices $u_{1}, u_{2}, u_{3}$, say $u_{1}$, is not a cut vertex of $\langle N(u)\rangle$. Hence, by Proposition 2.3, there is a Hamilton $\left(u, u_{1}\right)$-path $P^{\prime}=u P_{4} u_{1}$ of $\langle M(u)\rangle$. Then $N(u) \subseteq V(P)$ for the $(u, v)$-path $P=u P_{4} P_{1} v$.

Case 2. $\quad d(u, v)=1$. If $v$ is not a cut vertex of $H$ then, by Proposition 2.3, there exists a Hamilton $(u, v)$-path $P$ of $\langle M(u)\rangle$ and $N(u) \subseteq V(P)$.

Now let $v$ be a cut vertex of $H$. Then, by Proposition 2.2, $N(u)=A \cup B \cup\{v\}$ where $A \cap B=\emptyset, v \notin A \cup B$ and $\langle A\rangle,\langle B\rangle$ are complete graphs. Since $G$ is 3-connected, in $G-\{u, v\}$ there is a path $z_{2} P_{0} z_{1}$ such that $z_{1} \in A, z_{2} \in B$ and $V\left(P_{0}\right) \cap M(u)=\emptyset$. Consider a Hamilton $\left(z_{1}, z_{2}\right)$-path $P^{\prime}$ of $H$. Let $P^{\prime}=z_{1} P_{1} v b P_{2} z_{2}$, where $z_{1} P_{1}$ is a Hamilton path of $\langle A\rangle$ and $b P_{2} z_{2}$ is a Hamilton path of $\langle B\rangle$. Then the $(u, v)$-path $P=u b P_{2} z_{2} P_{0} z_{1} P_{1} v$ satisfies $N(u) \subseteq V(P)$.

So, in each case there exits a $(u, v)$-path $P$ with $N(u) \subseteq V(P)$. Consider a $(u, v)$-path $Q$ with $V(P) \subseteq V(Q)$ which has the maximum number of vertices from $N(v)$. Suppose that $N(v) \backslash V(Q) \neq \emptyset$ and $z \in N(v) \backslash V(Q)$. Clearly, $z v^{-} \notin E(G)$ where $v^{-}$is the predecessor of $v$ in $Q$. Then there exists $z_{1} \in N(v) \cap N(z) \cap N\left(v^{-}\right)$. Clearly, $z_{1} \in V(Q)$ (otherwise there is a $(u, v)$-path $Q^{\prime}=u \vec{Q} v^{-} z_{1} z v$ which satisfies $V(P) \subset V\left(Q^{\prime}\right)$ and $\left|N(v) \cap V\left(Q^{\prime}\right)\right|>|N(v) \cap V(Q)|$; a contradiction).

Since $N(u) \subseteq V(Q), z_{1} \neq u$. Hence $z_{1}^{-} z_{1}^{+} \in E(G)$ in $Q$ since $G$ is claw-free. Then there is a $(u, v)$-path $Q^{\prime}=u \vec{Q} z_{1}^{-} z_{1}^{+} \vec{Q} v^{-} z_{1} z v$ satisfying $V(P) \subset V\left(Q^{\prime}\right)$ and $\left|N(v) \cap V\left(Q^{\prime}\right)\right|>$ $|N(u) \cap V(Q)| ;$ a contradiction. So, $N(v) \cup N(v) \subseteq V(Q)$. 


\section{MAIN RESULT}

Theorem 3.1. Let $G$ be a 3-connected, locally connected, claw-free graph. Then for any pair of vertices $x, y$ with $d(x, y) \geq 3$ there is a Hamilton $(x, y)$-path of $G$.

Proof. Taking Theorem 2.5 into consideration, it is sufficient to prove that there is an $(x, y)$-path $Q$ with $N(x) \cup N(y) \subseteq V(Q)$.

Case 1. There is an $(x, y)$-path $x P_{0} y$ such that $\left|N(x) \cap V\left(P_{0}\right)\right|=\left|N(y) \cap V\left(P_{0}\right)\right|=1$, the unique vertex $x_{1} \in N(x) \cap V\left(P_{0}\right)$ is not a cut vertex of $\langle N(x)\rangle$ and the unique vertex $y_{1} \in N(y) \cap V\left(P_{0}\right)$ is not a cut vertex of $\langle N(y)\rangle$. Such a path we call a convenient $(x, y)$-path.

Then, by Proposition 2.3, there is a Hamilton $\left(x, x_{1}\right)$-path $x Q_{1} x_{1}$ of $\langle M(x)\rangle$ and a Hamilton $\left(y_{1}, y\right)$-path $y_{1} Q_{2} y$ of $\langle M(y)\rangle$. The path $Q=x Q_{1} P_{0} Q_{2} y$ satisfies the condition $N(x) \cup N(y) \subseteq V(Q)$.

Case 2. There does not exist a convenient $(x, y)$-path.

Since $G$ is 3-connected, there exist three $(x, y)$-paths $x P_{1} y, x P_{2} y, x P_{3} y$ such that $V\left(P_{i}\right) \cap$ $V\left(P_{j}\right)=\emptyset$ for $1 \leq i \leq j \leq 3$. We can assume that $\left|V\left(P_{i}\right) \cap N(x)\right|=\left|V\left(P_{i}\right) \cap N(y)\right|=1$ for $i=1,2,3$. Let $V\left(P_{i}\right) \cap N(x)=\left\{x_{i}\right\}$ and $V\left(P_{i}\right) \cap N(y)=\left\{y_{i}\right\}$.

Since $x P_{i} y$ is not a convenient $(x, y)$-path, either $x_{i}$ is a cut vertex of $\langle N(x)\rangle$ or $y_{i}$ is a cut vertex of $\langle N(y)\rangle, i=1,2,3$. This implies, by Proposition 2.2, that one of the graphs $\langle N(x)\rangle$ and $\langle N(y)\rangle$, say $\langle N(x)\rangle$, contains exactly two cut vertices and the other, $\langle N(y)\rangle$, contains at least one cut vertex. We assume that $y_{1}$ is a cut vertex of $\langle N(y)\rangle$ and $x_{2}, x_{3}$ are cut vertices of $\langle N(x)\rangle$. By Proposition 2.2, $x_{2} x_{3} \in E(G)$. Furthermore, $N(x)=A_{1} \cup A_{2}, N(y)=B_{1} \cup B_{2}$ where $A_{1} \cap A_{2}=\emptyset=B_{1} \cap B_{2}$ and $\left\langle A_{i}\right\rangle,\left\langle B_{i}\right\rangle$ are complete graphs for $i=1,2$. Without loss of generality we assume that $x_{1}, x_{2} \in$ $A_{1}, x_{3} \in A_{2}$ and $y_{1} \in B_{2}$. So, $\left|A_{1}\right| \geq 2,\left|A_{2}\right| \geq 2$ and $\left|B_{2}\right| \geq 2$. Let $P_{i}=Q_{i} v_{i} y_{i}$ for $i=1,2,3$. Then

$v_{1} z \notin E(G)$ for each $z \in N(y) \cap N\left(y_{1}\right)$ which is not a cut vertex of $\langle N(y)\rangle$.

(Otherwise we obtain a convenient $(x, y)$-path $\left.Q=x Q_{1} v_{1} z y\right)$. Let $u_{1} \in B_{1} \cap N\left(y_{1}\right)$. Since $G$ is claw-free, (2) implies that $u_{1}$ is adjacent to $v_{1}, u_{1}$ is the second cut vertex of $\langle N(y)\rangle$ and $\left|B_{1}\right| \geq 2$.

Subcase 2.1. $y_{2}$ and $y_{3}$ belong to different $B_{i}$ 's.

Then we can produce an $(x, y)$-path with $N(x) \cup N(y) \subseteq V(Q)$ in the following way. If $y_{2} \in B_{1}$ and $y_{3} \in B_{2}$ then

$$
Q=x\left[A_{2} \backslash\left\{x_{3}\right\}\right] x_{3} P_{3} y_{3}\left[B_{2} \backslash\left\{y_{1}, y_{3}\right\}\right] y_{1} v_{1} \bar{Q}_{1} x_{1}\left[A_{1} \backslash\left\{x_{1}, x_{2}\right\}\right] x_{2} P_{2} y_{2}\left[B_{1} \backslash\left\{u_{1}, y_{2}\right\}\right] u_{1} y
$$

If $y_{2} \in B_{2}$ and $y_{3} \in B_{1} \backslash\left\{u_{1}\right\}$ then

$$
Q=x\left[A_{2} \backslash\left\{x_{3}\right\}\right] x_{3} P_{3} y_{3}\left[B_{1} \backslash\left\{u_{1}, y_{3}\right\}\right] u_{1} v_{1} \overleftarrow{Q}_{1} x_{1}\left[A_{1} \backslash\left\{x_{1}, x_{2}\right\}\right] x_{2} P_{2} y_{2}\left[B_{2} \backslash\left\{y_{2}\right\}\right] y
$$

If $y_{2} \in B_{2}, y_{3}=u_{1}$ and $v_{3} z \in E(G)$ for some $z \in B_{1} \backslash\left\{u_{1}\right\}$ then by considering the path $P_{3}^{\prime}=Q_{3} v_{3} z$ instead $P_{3}$ will obtain the previous situation.

Now let $y_{2} \in B_{2}, y_{3}=u_{1}$ and $N\left(v_{3}\right) \cap B_{1}=\left\{u_{1}\right\}$. This implies that $v_{3} y_{1} \in E(G)$ because otherwise the set $\left\{y_{1}, u_{1}, v_{3}, g\right\}$ induces $K_{1,3}$ where $g \in B_{1} \backslash\left\{u_{1}\right\}$. Then

$$
Q=x\left[A_{2} \backslash\left\{x_{3}\right\}\right] x_{3} Q_{3} v_{3} y_{1}\left[B_{2} \backslash\left\{y_{1}, y_{2}\right\}\right] y_{2} \stackrel{\leftarrow}{P}_{2} x_{2}\left[A_{1} \backslash\left\{x_{1}, x_{2}\right\}\right] x_{1} Q_{1} v_{1} y_{3}\left[B_{1} \backslash\left\{y_{3}\right\}\right] y
$$


Subcase 2.2. $y_{2}$ and $y_{3}$ belong to the same $B_{i}$. If $y_{2}, y_{3} \in B_{2}$ then the path

$$
Q=x\left[A_{2} \backslash\left\{x_{3}\right\}\right] x_{3} P_{3} y_{3}\left[B_{2} \backslash\left\{y_{2}, y_{3}\right\}\right] y_{2} \bar{P}_{2} x_{2}\left[A_{1} \backslash\left\{x_{1}, x_{2}\right\}\right] x_{1} Q_{1} v_{1} u_{1}\left[B_{1} \backslash\left\{u_{1}\right\}\right] y
$$

satisfies the condition $N(x) \cup N(y) \subseteq V(Q)$. If $y_{2}, y_{3} \in B_{1}$ then by considering $u_{1}$ instead $y_{1}$ we will obtain the same situation since $v_{1} u_{1}, v_{1} y_{1} \in E(G)$.

Theorem 3.2. Let $G$ be a 3-connected, locally connected, claw-free graph. Then for each pair of adjacent vertices $x, y$ there is a Hamilton $(x, y)$-path.

Proof. If for some $v \in\{x, y\}, N(v) \cap N\left(w_{1}\right) \cap N\left(w_{2}\right) \neq \emptyset$ for each pair of non-adjacent vertices $w_{1}, w_{2} \in N(v)$ then, by Theorem 2.6, there is a Hamilton $(x, y)$-path. Suppose now that there exist non-adjacent vertices $z_{1}, z_{2} \in N(x)$ and non-adjacent vertices $v_{1}, v_{2} \in N(y)$ such that $N(x) \cap N\left(z_{1}\right) \cap N\left(z_{2}\right)=\emptyset=N(y) \cap N\left(v_{1}\right) \cap N\left(v_{2}\right)$. Then, by Proposition 2.1, $N(x)=A_{1} \cup A_{2}$ and $N(y)=B_{1} \cup B_{2}$ where $A_{1} \cap A_{2}=\emptyset=B_{1} \cap B_{2},\left|A_{i}\right| \geq 2,\left|B_{i}\right| \geq 2$ and $\left\langle A_{i}\right\rangle,\left\langle B_{i}\right\rangle$ are complete graphs for $i=1,2$. Without loss of generality we assume that $y \in A_{1}$. Suppose there does not exist a Hamilton $(x, y)$-path of $G$. Then, by Theorem 2.5 ,

$$
\text { there does not exist an }(x, y)-\text { path } Q \text { with } N(x) \cup N(y) \subseteq V(Q) .
$$

Case 1. $y$ is not a cut vertex of $\langle N(x)\rangle$.

By Proposition 2.3, there is a Hamilton $(x, y)$-path of $\langle M(x)\rangle$. Then (3) implies that $N(y) \backslash M(x) \neq \emptyset$ and the vertex $x$ and the set $N(y) \backslash M(x)$ are in different $B_{i}$ 's in $N(y)$. Without loss of generality we assume that $x \in B_{2}$ and $N(y) \backslash M(x) \subseteq B_{1}$. Since $\langle N(y)\rangle$ is connected,

$$
\text { there exists an edge } z u \text { with } u \in N(x) \backslash\{y\} \text { and } z \in N(y) \backslash M(x) .
$$

Furthermore,

there does not exist an edge $z u$ such that

$$
z \in N(y) \backslash M(x), u \in N(x) \backslash\{y\} \text { and } u \text { is not a cut vertex of }\langle N(x)\rangle \text {. }
$$

Assuming the contrary, we can produce a path $Q$ contradicting (3) in the following way. Let $a_{1} a_{2}$ be an edge such that $a_{1} \in A_{1}, a_{2} \in A_{2}$ and $u \notin\left\{a_{1}, a_{2}\right\}$.

If $u \in A_{1}$ and $y \neq a_{1}$ then

$$
Q=x\left[A_{2} \backslash\left\{a_{2}\right\}\right] a_{2} a_{1}\left[A_{1} \backslash\left\{a_{1}, y, u\right\}\right] u z[(N(y) \backslash M(x)) \backslash\{z\}] y .
$$

If $u \in A_{2}$ and $y \neq a_{1}$ then

$$
Q=x\left[A_{1} \backslash\left\{y, a_{1}\right\}\right] a_{1} a_{2}\left[A_{2} \backslash\left\{u, a_{2}\right\}\right] u z[(N(y) \backslash M(x)) \backslash\{z\}] y .
$$

Now suppose that $y$ is the only choice for $a_{1}$. Then $\{u, y\}$ is a cut set of $\langle N(x)\rangle$. If $u \in A_{1}$ then $u$ has a neighbour $v$ in $A_{2}$ and (3) implies that there is a vertex $s \in A_{1} \backslash\{u, y\}$.

If $s \in B_{1}$ then $Q=x\left[A_{2} \backslash\{v\}\right] v u z[(N(y) \backslash M(x)) \backslash\{z\}] s\left[A_{1} \backslash\{s, y, u\}\right] y$. If $s \in B_{2}$ and $a_{2} \in B_{1}$ then

$$
Q=x\left[A_{2} \backslash\left\{a_{2}\right\}\right] a_{2}[(N(y) \backslash M(x)) \backslash\{z\}] z u\left[A_{1} \backslash\{y, u\}\right] y .
$$

If $s \in B_{2}$ and $a_{2} \in B_{2}$ then $s a_{2} \in E(G)$ and

$$
Q=x\left[A_{2} \backslash\left\{a_{2}\right\}\right] a_{2} s\left[A_{1} \backslash\{s, y, u\}\right] u z[(N(y) \backslash M(x)) \backslash\{z\}] y .
$$


In each case we obtained a contradiction to (3). So, (5) is proved.

Now consider an edge $z u$ with $u \in N(x) \backslash\{y\}$ and $z \in N(y) \backslash M(x)$. Then, by (5), $u$ is a cut vertex of $\langle N(x)\rangle$. (Note that if $u \in A_{1}$ then (3) implies $\left|A_{1}\right| \geq 3$ ). Choose vertices $g_{1} \in N(u) \cap\left(A_{1} \backslash\{y\}\right)$ and $g_{2} \in N(u) \cap A_{2}$. Then $E(G) \cap\left\{z_{g_{1}}, z_{g_{2}}\right\} \neq \emptyset$ since $G$ is claw-free. This and (5) imply that $\langle N(x)\rangle$ has two cut vertices, $u_{1}$ and $u_{2}$, and $N(z) \cap(N(x) \backslash\{y\})=\left\{u_{1}, u_{2}\right\}$ for each vertex $z \in N(y) \backslash M(x)$ having neighbours in $N(x) \backslash\{y\}$. The last property and (3) imply that $\left|N\left(u_{1}\right) \cap(N(y) \backslash M(x))\right|=1$.

Let $N\left(u_{1}\right) \cap(N(y) \backslash M(x))=\left\{z_{0}\right\}$. Clearly, (3) implies that $|N(y) \backslash M(x)| \geq 2$. Since $G$ is 3-connected, in $G-\left\{z_{0}, y\right\}$ there exists a path $s_{1} P_{0} s_{2}$ such that $s_{2} \in N(y) \backslash(M(x) \cup$ $\left.\left\{z_{0}\right\}\right), s_{1} \in N(x) \backslash\{y\}$ and $V\left(P_{0}\right) \cap(N(x) \cup N(y))=\emptyset$. But now we can indicate an $(x, y)$-path $Q$ with $N(x) \cup N(y) \subseteq V(Q)$, contradicting (3).

If $s_{1} \in A_{2}$ then

$$
Q=x\left[A_{2} \backslash\left\{s_{1}\right\}\right] s_{1} P_{0} s_{2}\left[(N(y) \backslash M(x)) \backslash\left\{z_{0}, s_{2}\right\}\right] z_{0} u_{1}\left[A_{1} \backslash\left\{u_{1}, y\right\}\right] y .
$$

If $s_{1} \in A_{1}$ then $Q=x\left[A_{2} \backslash\left\{u_{2}\right\}\right] u_{2} z_{0}\left[(N(y) \backslash M(x)) \backslash\left\{z_{0}, s_{2}\right\}\right] s_{2} \overleftarrow{P}_{0} s_{1}\left[A_{1} \backslash\left\{s_{1}, y\right\}\right] y$.

Remark 1. By using the same argument, we will obtain a contradiction in the case when $x$ is not a cut vertex of $\langle N(y)\rangle$.

Case 2. $x$ is a cut vertex of $\langle N(y)\rangle$ and $y$ is a cut vertex of $\langle N(x)\rangle$.

By Proposition 2.1, $\left|A_{1}\right| \geq 2$. Hence, $N(x) \cap N(y) \supseteq A_{1} \backslash\{y\} \neq \emptyset$. Without loss of generality we assume that $A_{1} \cap B_{1} \neq \emptyset$. Then $A_{1} \backslash\{y\} \subseteq B_{1}$ and $A_{1} \cap B_{2}=\emptyset$. Furthermore, $A_{2} \cap B_{2} \neq \emptyset$ because $x$ is a cut vertex of $\langle N(y)\rangle$.

Since $G$ is 3-connected, in $G-\{x, y\}$ there exists a path $g_{2} P_{0} g_{1}$ such that $g_{2} \in A_{2}, g_{1} \in$ $A_{1}$ and $V\left(P_{0}\right) \cap M(x)=\emptyset$. Now we shall produce an $(x, y)$-path $Q$ with $N(x) \cup N(y) \subseteq$ $V(Q)$, contradicting (3).

If $x \in B_{2}$ then $B_{2} \subseteq A_{2} \cup\{x\}$ and $Q=x\left[A_{2} \backslash\left\{g_{2}\right\}\right] g_{2} P_{0} g_{1}\left[B_{1} \backslash V\left(P_{0}\right)\right] y$.

Now let $x \in B_{1}$. Then $B_{1}=\left(A_{1} \backslash\{y\}\right) \cup\{x\}$. Choose a vertex $a \in A_{2} \cap B_{2}$. If $V\left(P_{0}\right) \cap\left(B_{2} \backslash A_{2}\right)=\emptyset$ then $Q=x\left[A_{1} \backslash\left\{g_{1}, y\right\}\right] g_{1} \overleftarrow{P}_{0} g_{2}\left[A_{2} \backslash\left\{a, g_{2}\right\}\right] a\left[B_{2} \backslash A_{2}\right] y$. If $V\left(P_{0}\right) \cap\left(B_{2} \backslash A_{2}\right) \neq \emptyset$ and $b$ is the last common vertex of $P_{0}$ and $B_{2} \backslash A_{2}$ then

$$
Q=x\left[A_{2} \backslash\{a\}\right] a\left[B_{2} \backslash\left(A_{2} \cup\{b\}\right)\right] b \vec{P}_{0} g_{1}\left[A_{1} \backslash\left\{g_{1}, y\right\}\right] y .
$$

In each case we obtained a contradiction to (3). So, there exists a Hamilton $(x, y)$-path of $G$. The proof of the theorem is complete.

Theorem 3.3. Let $G$ be a 3-connected, locally connected, claw-free graph. Then for any pair of vertices $x, y$ with $d(x, y)=2$ there is a Hamilton $(x, y)$-path of $G$.

Proof. If for some $v \in\{x, y\}, N(v) \cap N\left(w_{1}\right) \cap N\left(w_{2}\right) \neq \emptyset$ for each pair of nonadjacent vertices $w_{1}, w_{2} \in N(v)$, then, by Theorem 2.6, there is a Hamilton $(x, y)$-path of $G$. Suppose now that there exist non-adjacent vertices $z_{1}, z_{2} \in N(x)$ and non-adjacent vertices $y_{1}, y_{2} \in N(y)$ such that

$$
N(x) \cap N\left(z_{1}\right) \cap N\left(z_{2}\right)=\emptyset=N(y) \cap N\left(y_{1}\right) \cap N\left(y_{2}\right) .
$$

By Proposition 2.1, $N(x)=A_{1} \cup A_{2}, N(y)=B_{1} \cup B_{2}$, where $A_{1} \cap A_{2}=\emptyset=B_{1} \cap$ $B_{2},\left|A_{i}\right| \geq 2,\left|B_{i}\right| \geq 2$ and $\left\langle A_{i}\right\rangle,\left\langle B_{i}\right\rangle$ are complete graphs for $i=1,2$. Taking Theorem 2.5 into consideration, it is sufficient to prove that there exists an $(x, y)$-path $Q$ with $N(x) \cup N(y) \subseteq V(Q)$. Without loss of generality we assume that $A_{1} \cap B_{1} \neq \emptyset$. 
Case 1. $A_{1} \cap B_{2} \neq \emptyset$ or $B_{1} \cap A_{2} \neq \emptyset$.

We assume that $A_{1} \cap B_{2} \neq \emptyset$. Let $u_{i} \in A_{1} \cap B_{i}$ for $i=1,2$.

Subcase 1.1. One of the sets $A_{1} \cap B_{1}, A_{1} \cap B_{2}$ contains a cut vertex of $\langle N(x)\rangle$.

Let, for example, $u_{2}$ be a cut vertex of $\langle N(x)\rangle$ and $q \in N\left(u_{2}\right) \cap A_{2}$. If $\left|A_{1} \cap B_{1}\right| \geq 2$ and $u_{3} \in\left(A_{1} \cap B_{2}\right) \backslash\left\{u_{2}\right\}$ then the following path $Q$ includes $N(x) \cup N(y): Q=x\left[A_{2} \backslash\right.$ $\{g\}] g u_{2}\left[B_{2} \backslash N(x)\right] u_{3}\left[A_{1} \backslash\left\{u_{1}, u_{2}, u_{3}\right\}\right] u_{1}\left[B_{1} \backslash N(x)\right] y$. Now let $A_{1} \cap B_{2}=\left\{u_{2}\right\}$. We shall show that there is a vertex $v_{0} \in B_{2} \backslash\left\{u_{2}\right\}$ such that $N\left(v_{0}\right) \cap A_{2} \neq \emptyset$. Assuming the contrary, we obtain that $B_{2} \cap A_{2}=\emptyset$. Furthermore, $v u_{1} \in E(G)$ for each $v \in B_{2} \backslash\left\{u_{2}\right\}$, because $G$ is claw-free and $u_{2}$ is a cut vertex of $\langle N(x)\rangle$. But then $u_{1} \in N(y) \cap N\left(w_{1}\right) \cap N\left(w_{2}\right)$ for each pair of non-adjacent vertices $w_{1}, w_{2} \in N(y)$, which contradicts (6).

So, there are vertices $z_{0} \in A_{2}$ and $v_{0} \in B_{2} \backslash\left\{u_{2}\right\}$ which are adjacent. Then the following path $Q$ includes $N(x) \cup N(y)$ :

$$
Q=x\left[A_{2} \backslash\left\{z_{0}\right\}\right] z_{0} v_{0}\left[B_{2} \backslash\left\{u_{2}, v_{0}\right\}\right] u_{2}\left[A_{1} \backslash\left\{u_{1}, u_{2}\right\}\right] u_{1}\left[B_{1} \backslash N(x)\right] y .
$$

Subcase 1.2. $A_{1} \cap B_{1}$ and $A_{1} \cap B_{2}$ contain no cut vertex of $\langle N(x)\rangle$ and $\left|A_{1} \cap B_{j}\right| \geq 2$ for some $j \in\{1,2\}$.

Let $u_{0}, u_{1} \in A_{1} \cap B_{1}$ and $u_{2} \in B_{2} \cap A_{1}$. Clearly, there is an edge $a_{1} a_{2}$ such that $a_{1} \in A_{1} \backslash\left\{u_{2}\right\}$ and $a_{2} \in A_{2}$. Then there is an $(x, y)$-path $Q$,

$$
Q=x\left[A_{2} \backslash\left\{a_{2}\right\}\right] a_{2} R\left[A_{1} \backslash\left\{u_{0}, u_{1}, u_{2}, a_{1}\right\}\right] u_{2}\left[B_{2} \backslash N(x)\right] y
$$

with $N(x) \cup N(y) \subseteq V(Q)$ where

$$
R= \begin{cases}a_{1} u_{0}\left[B_{1} \backslash N(x)\right] u_{1} & \text { if } a_{1} \notin\left\{u_{0}, u_{1}\right\} \\ a_{1}\left[B_{1} \backslash N(x)\right] u_{1} & \text { if } a_{1}=u_{0} \\ a_{1}\left[B_{1} \backslash N(x)\right] u_{0} & \text { if } a_{1}=u_{1}\end{cases}
$$

Subcase 1.3. $A_{1} \cap B_{i}=\left\{u_{i}\right\}$ and $u_{i}$ is not a cut vertex of $\langle N(x)\rangle$ for $i=1,2$.

First we consider the situation when $A_{2} \cap\left(B_{1} \cup B_{2}\right) \neq \emptyset$. W.l.o.g. we assume that $A_{2} \cap$ $B_{2} \neq \emptyset$. Let $s \in A_{2} \cap B_{2}$. Then the following $(x, y)$-path $Q$ includes the set $N(x) \cup N(y)$ :

$$
Q=x\left[A_{2} \backslash\{s\}\right] s\left[\left(B_{2} \backslash A_{2}\right) \backslash\left\{u_{2}\right\}\right] u_{2}\left[A_{1} \backslash\left\{u_{1}, u_{2}\right\}\right] u_{1}\left[\left(B_{1} \backslash A_{2}\right) \backslash\left\{u_{1}\right\}\right] y .
$$

Now let $A_{2} \cap\left(B_{1} \cup B_{2}\right)=\emptyset$. Since $G$ is 3-connected, in $G-\left\{u_{1}, u_{2}\right\}$ there is an $(x, y)$-path $x s_{1} P s_{2} y$, where $s_{1} \in N(x), s_{2} \in N(y)$ and $V(P) \cap(N(x) \cup N(y))=\emptyset$. W.1.o.g. we assume that $s_{2} \in B_{2}$. Clearly, there is an edge $a_{1} a_{2}$ with $a_{1} \in A_{1} \backslash\left\{u_{1}\right\}$ and $a_{2} \in A_{2}$. Now we will produce an $(x, y)$-path $Q$ with $N(x) \cup N(y) \subseteq V(Q)$.

If $s_{1} \in A_{2}$ then

$$
Q=x\left[A_{2} \backslash\left\{s_{1}\right\}\right] s_{1} P s_{2}\left[B_{2} \backslash\left\{s_{2}, u_{2}\right\}\right] u_{2}\left[A_{1} \backslash\left\{u_{1}, u_{2}\right\}\right] u_{1}\left[B_{1} \backslash\left\{u_{1}\right\}\right] y .
$$

If $s_{1} \in A_{1}$ then $Q=x\left[A_{2} \backslash\left\{a_{2}\right\}\right] a_{2} R\left[A_{1} \backslash\left\{a_{1}, u_{1}, u_{2}, s_{1}\right\}\right] u_{1}\left[B_{1} \backslash\left\{u_{1}\right\}\right] y$, where

$$
R= \begin{cases}a_{1} u_{2}\left[B_{2} \backslash\left\{u_{2}, s_{2}\right\}\right] s_{2} \overleftarrow{P} s_{1} & \text { if } a_{1} \notin\left\{s_{1}, u_{2}\right\} \\ a_{1}\left[B_{2} \backslash\left\{a_{1}, s_{2}\right\}\right] s_{2} \widetilde{P} s_{1} & \text { if } a_{1}=u_{2} \\ a_{1} P s_{2}\left[B_{2} \backslash\left\{u_{2}, s_{2}\right\}\right] u_{2} & \text { if } a_{1}=s_{1}\end{cases}
$$

Remark 2. By symmetry, the case $B_{1} \cap A_{2} \neq \emptyset$ requires the same argument but for sets $B_{1} \cap A_{1}$ and $B_{1} \cap A_{2}$. 
Case 2. $A_{1} \cap B_{2}=B_{1} \cap A_{2}=\emptyset$ and also $A_{2} \cap B_{2}=\emptyset$. Then

$A_{1} \cap B_{1}$ contains a vertex $u_{1}$ which is not a cut vertex of $\langle N(x)\rangle$.

This is evident if $\left|A_{1} \cap B_{1}\right| \geq 2$. If $\left|A_{1} \cap B_{1}\right|=1$ then (7) follows from the fact that $G$ is claw-free. Clearly, (7) implies that there is an edge $a_{1} a_{2}$ such that $a_{1} \in A_{1} \backslash\left\{u_{1}\right\}$ and $a_{2} \in A_{2}$. Furthermore, we have that $\langle N(y)\rangle$ is connected.

If there exists an edge $v_{1} v_{2}$ with $v_{1} \in B_{1} \backslash A_{1}$ and $v_{2} \in B_{2}$ then there is an $(x, y)$ path $Q=x\left[A_{2} \backslash\left\{a_{2}\right\}\right] a_{2} a_{1}\left[A_{1} \backslash\left\{a_{1}, u_{1}\right\}\right] u_{1}\left[B_{1} \backslash\left(N(x) \cup\left\{v_{1}\right\}\right)\right] v_{1} v_{2}\left[B_{2} \backslash\left\{v_{2}\right\}\right] y$ with $N(x) \cup N(y) \subseteq V(Q)$.

Suppose now that $v_{1} \in A_{1}$ for each edge $v_{1} v_{2}$ with $v_{1} \in B_{1}$ and $v_{2} \in B_{2}$ and consider one of these edges, $v_{1} v_{2}$. Clearly, $B_{1} \subseteq A_{1}$. (Otherwise a set $\left\{x, v_{1}, v_{2}, g_{1}\right\}$ induces $K_{1,3}$, where $\left.g_{1} \in\left(B_{1} \backslash A_{1}\right) \cap N\left(v_{1}\right)\right)$. If $v_{1}$ is not a cut vertex of $\langle N(x)\rangle$ then there is an edge $w_{1} w_{2}$ such that $w_{1} \in A_{1} \backslash\left\{v_{1}\right\}$ and $w_{2} \in A_{2}$. Then the path $Q=x\left[A_{2} \backslash\left\{w_{2}\right\}\right] w_{2} w_{1}\left[A_{1} \backslash\right.$ $\left.\left\{w_{1}, v_{1}\right\}\right] v_{1} v_{2}\left[B_{2} \backslash\left\{v_{2}\right\}\right] y$ satisfies the condition $N(x) \cup N(y) \subseteq V(Q)$.

Now we assume that $v_{1}$ is a cut vertex of $\langle N(x)\rangle$. Let $s_{0} \in N\left(v_{1}\right) \cap A_{2}$. Then $v_{2} s_{0} \in$ $E(G)$. (Otherwise $v_{2} z \in E(G)$ for each $z \in B_{1}$ since $G$ is claw-free. But then $v_{2}$ $\in N(y) \cap N\left(b_{1}\right) \cap N\left(b_{2}\right)$ for each pair of non-adjacent vertices $b_{1}, b_{2} \in N(y)$, which contradicts (6)).

Subcase 2.1. $s_{0}$ is not a cut vertex of $N(x)$. Then there is an edge $v_{1} a_{0}$ with $a_{0} \in$ $A_{2} \backslash\left\{s_{0}\right\}$ and an $(x, y)$-path $Q=x\left[A_{1} \backslash\left\{v_{1}\right\}\right] v_{1} a_{0}\left[A_{2} \backslash\left\{a_{0}, s_{0}\right\}\right] s_{0} v_{2}\left[B_{2} \backslash\left\{v_{2}\right\}\right] y$ with $N(x) \cup N(y) \subseteq V(Q)$.

Subcase 2.2. $v_{1}$ is not a cut vertex of $\langle N(y)\rangle$.

Clearly, there is an edge $b_{1} b_{2}$ such that $b_{1} \in B_{1} \backslash\left\{v_{1}\right\}$ and $b_{2} \in B_{2}$. Then there is an $(x, y)$-path $Q=x\left[A_{2} \backslash\left\{s_{0}\right\}\right] s_{0} v_{1}\left[A_{1} \backslash\left\{v_{1}, b_{1}\right\}\right] b_{1} b_{2}\left[B_{2} \backslash\left\{b_{2}\right\}\right] y$ with $N(x) \cup N(y) \subseteq V(Q)$.

Subcase 2.3. $\quad v_{2}$ is not a cut vertex of $\langle N(y)\rangle$.

Then there is an edge $v_{1} v_{3}$, where $v_{3} \in B_{2} \backslash\left\{v_{2}\right\}$. By (7), the set $A_{1} \cap B_{1}$ contains a vertex $u_{1}$ which is not a cut vertex of $\langle N(x)\rangle$. Clearly, $u_{1} \neq v_{1}$. Then there is an $(x, y)$-path $Q=x\left[A_{2} \backslash\left\{s_{0}\right\}\right] s_{0} v_{2}\left[B_{2} \backslash\left\{v_{2}, v_{3}\right\}\right] v_{3} v_{1}\left[A_{1} \backslash\left\{v_{1}, u_{1}\right\}\right] u_{1} y$ with $N(x) \cup N(y) \subseteq V(Q)$.

Subcase 2.4. $\langle N(y)\rangle$ has two cut vertices, $v_{1}$ and $v_{2}$, and $\langle N(x)\rangle$ has two cut vertices, $v_{1}$ and $s_{0}$.

Since $G$ is 3-connected, in $G-\left\{x, s_{0}\right\}$ there is a path $s_{1} P s_{2}$ where $s_{1} \in A_{2}, s_{2} \in$ $N(y) \cup A_{1}$ and $V(P) \cap(N(x) \cup N(y))=\emptyset$. Then we can produce an $(x, y)$-path $Q$ with $N(x) \cup N(y) \subseteq V(Q)$ in the following way.

If $s_{2} \in B_{2}$ then $Q=x\left[A_{1} \backslash\left\{v_{1}\right\}\right] v_{1} s_{0}\left[A_{2} \backslash\left\{s_{0}, s_{1}\right\}\right] s_{1} P s_{2}\left[B_{2} \backslash\left\{s_{2}\right\}\right] y$ and if $s_{2} \in A_{1}$ then $Q=x\left[A_{1} \backslash\left\{s_{2}\right\}\right] s_{2} \stackrel{\leftarrow}{P} s_{1}\left[A_{2} \backslash\left\{s_{1}, s_{0}\right\}\right] s_{0} v_{2}\left[B_{2} \backslash\left\{v_{2}\right\}\right] y$.

Case 3. $A_{1} \cap B_{2}=A_{2} \cap B_{1}=\emptyset$ and $A_{2} \cap B_{2} \neq \emptyset$.

Subcase 3.1. For each $i \in\{1,2\}$ the set $A_{i} \cap B_{i}$ contains a vertex $u_{i}$ which is not a cut vertex of $\langle N(x)\rangle$.

(a) $\left\{u_{1}, u_{2}\right\}$ is a cut set of $\langle N(x)\rangle$. Then there is an edge $a_{1} u_{2}$ where $a_{1} \in A_{1} \backslash\left\{u_{1}\right\}$ and an edge $u_{1} a_{2}$ where $a_{2} \in A_{2} \backslash\left\{u_{2}\right\}$. Consider the set $\left\{a_{1}, a_{2}, y, u_{2}\right\}$. Since $\left\{u_{1}, u_{2}\right\}$ is a cut set of $\langle N(x)\rangle, a_{1} a_{2} \notin E(G)$. Then $y a_{1} \in E(G)$ or $y a_{2} \in E(G)$ because $G$ is claw-free. Since these situations are similar, we consider the case $y a_{1} \in E(G)$ only. We have that $a_{1} \in A_{1}$ and $A_{1} \cap B_{2}=\emptyset$. Therefore $a_{1} \in B_{1}$. Then

$$
Q=x\left[A_{1} \backslash\left\{a_{1}, u_{1}\right\}\right] a_{1}\left[B_{1} \backslash\left\{u_{1}\right\}\right] u_{1} a_{2}\left[A_{2} \backslash\left\{a_{2}, u_{2}\right\}\right] u_{2}\left[B_{2} \backslash\left\{u_{2}\right\}\right] y
$$

is an $(x, y)$-path $Q$ with $N(x) \cup N(y) \subseteq V(Q)$. 
(b) $\left\{u_{1}, u_{2}\right\}$ is not a cut set of $\langle N(x)\rangle$.

Then there is an edge $a_{1} a_{2}$ where $a_{i} \in A_{i} \backslash\left\{u_{i}\right\}$ for $i=1,2$. If one of the sets $A_{j} \cap B_{j}, 1 \leq j \leq 2$, say $A_{1} \cap B_{1}$, contains a vertex $u_{0} \notin\left\{u_{1}, u_{2}\right\}$, then the path $Q=$ $x u_{0}\left[B_{1} \backslash A_{1}\right] u_{1}\left[A_{1} \backslash\left\{u_{0}, u_{1}, a_{1}\right\}\right] a_{1} a_{2}\left[A_{2} \backslash\left\{a_{2}, u_{2}\right\}\right] u_{2}\left[B_{2} \backslash A_{2}\right] y$ satisfies the condition $N(x) \cup N(y) \subseteq V(Q)$.

Now let $A_{i} \cap B_{i}=\left\{u_{i}\right\}$ for $i=1,2$. Since $G$ is 3-connected, in $G-\left\{u_{1}, u_{2}\right\}$ there is an $(y, x)$-path $y s_{1} P s_{2} x$, where $s_{1} \in N(y) \backslash\left\{u_{1}, u_{2}\right\}, s_{2} \in N(x) \backslash\left\{u_{1}, u_{2}\right\}$ and $V(P) \cap$ $(N(x) \cup N(y))=\emptyset$. We assume that $s_{1} \in B_{2}$. Now we will produce an $(x, y)$-path $Q$ satisfying the condition $N(x) \cup N(y) \subseteq V(Q)$. If $s_{2} \in A_{1}$ then $Q=x\left[A_{2} \backslash\left\{u_{2}\right\}\right] u_{2}\left[B_{2} \backslash\right.$ $\left\{u_{2}, s_{1}\right\} s_{1} P s_{2}\left[A_{1} \backslash\left\{s_{2}, u_{1}\right\}\right] u_{1}\left[B_{1} \backslash\left\{u_{1}\right\}\right] y$.

If $s_{2} \in A_{2}$ and $a_{2} \neq s_{2}$ then

$$
Q=x\left[A_{2} \backslash\left\{s_{2}, a_{2}, u_{2}\right\}\right] u_{2}\left[B_{2} \backslash\left\{u_{2}, s_{1}\right\}\right] s_{1} P s_{2} a_{2} a_{1}\left[A \backslash\left\{a_{1}, u_{1}\right\}\right] u_{1}\left[B_{1} \backslash\left\{u_{1}\right\}\right] y .
$$

The same path, but with $a_{2}$ deleted, corresponds to the case $s_{2} \in A_{2}$ and $s_{2}=a_{2}$.

Subcase 3.2. For some $i \in\{1,2\}$ the set $A_{i} \cap B_{i}$ consists of the unique vertex $u_{i}$ which is a cut vertex of $\langle N(x)\rangle$.

We assume that $A_{1} \cap B_{1}=\left\{u_{1}\right\}$ and $u_{1}$ is a cut vertex of $\langle N(x)\rangle$. Let $u_{2}$ be a vertex from $A_{2} \cap N\left(u_{1}\right)$. Since $G$ is claw-free, $y u_{2} \in E(G)$. So, $u_{2} \in B_{2}$. Then $v_{1} z_{1} \in E(G)$ for some $v_{1} \in A_{1} \backslash\left\{u_{1}\right\}$ and $z_{1} \in B_{1} \backslash\left\{u_{1}\right\}$. (Otherwise $z u_{2} \in E(G)$ for each $z \in B_{1}$ since $G$ is claw-free. Therefore, $u_{2} \in N(y) \cap N\left(w_{1}\right) \cap N\left(w_{2}\right)$ for each pair of non-adjacent vertices $w_{1}, w_{2} \in N(y)$, which contradicts (6)).

If $N\left(u_{1}\right) \cap A_{2}=\left\{u_{2}\right\}$ then $u_{2}$ is a cut vertex of $\langle N(x)\rangle$. Hence, by symmetry, $v_{2} z_{2} \in$ $E(G)$ for some $v_{2} \in A_{2} \backslash\left\{u_{2}\right\}$ and $z_{2} \in B_{2} \backslash\left\{u_{2}\right\}$. Then $N(x) \cup N(y) \subseteq V(Q)$ for an $(x, y)$-path $Q$,

$$
Q=x\left[A_{1} \backslash\left\{v_{1}, u_{1}\right\}\right] v_{1} z_{1}\left[B_{1} \backslash\left\{u_{1}, z_{1}\right\}\right] u_{1} u_{2}\left[A_{2} \backslash\left\{u_{2}, v_{2}\right\}\right] v_{2} z_{2}\left[B_{2} \backslash\left\{z_{2}, u_{2}\right\}\right] y .
$$

Now let $\left|N\left(u_{1}\right) \cap A_{2}\right| \geq 2, u_{3} \in N\left(u_{1}\right) \cap A_{2}$ and $u_{3} \neq u_{2}$. Then for an $(x, y)$-path

$$
Q=x\left[A_{1} \backslash\left\{u_{1}, v_{1}\right\}\right] v_{1} z_{1}\left[B_{1} \backslash\left\{u_{1}, z_{1}\right\}\right] u_{1} u_{3}\left[A_{2} \backslash\left\{u_{2}, u_{3}\right\}\right] u_{2}\left[B_{2} \backslash A_{2}\right] y
$$

the condition $N(x) \cup N(y) \subseteq V(Q)$ holds. The proof of Theorem 3.3 is complete.

Theorem 3.4. A locally connected, claw-free graph $G$ with $|V(G)| \geq 4$ is Hamiltonconnected if and only if $G$ is 3-connected.

Proof. Clearly, if $G$ is Hamilton-connected and has at least 4 vertices then it is also 3-connected. Conversely, if $G$ is a 3-connected, claw-free graph with $|V(G)| \geq 4$ then it follows from Theorems 3.1-3.3 that $G$ is Hamilton-connected.

\section{ACKNOWLEDGMENTS}

I thank R. Häggkvist and T. Denley for useful comments. I also thank the referees for their remarks and suggestions which led to this improved version. 


\section{References}

[1] J. A. Bondy and U. S. R. Murty, Graph Theory with Applications, Macmillan, London and Elsevier, New York (1976).

[2] H. J. Broersma and H. J. Veldman, 3-connected line graphs of triangular graphs are panconnected and 1-hamiltonian, J. Graph Theory 11 (1987), 399-407.

[3] G. Chartrand, R. T. Gould, and A. D. Polimeni, A note on locally connected and Hamiltonianconnected graphs, Israel J. Math. 33 (1979), 5-8.

[4] G. Chartrand and R. E. Pippert, Locally connected graphs, Casopis Pest. Mat. 99 (1974) 158 163.

[5] V. Chvátal and P. Erdös, A note on Hamiltonian circuits, Discrete Math. 2 (1972), 111-113.

[6] L. Clark, Hamiltonian properties of connected locally connected graphs. Congr. Numer. 32 (1981), 199-204.

[7] S. V. Kanetkar and P. R. Rao, Connected, locally 2-connected, $K_{1,3}$-free graphs are panconnected, J. Graph Theory 8 (1984), 347-353.

[8] D. J. Oberly and D. P. Sumner, Every connected, locally connected nontrivial graph with no induced claw is Hamiltonian, J. Graph Theory 3 (1979), 351-356.

[9] H. Whitney, Congruent graphs and the connectivity of graphs, Amer. J. Math. 54 (1932), 150168.

Received October 18, 1994 